\title{
Hyperglycemia During the First Three Days of Life Increases the Risk of Retinopathy of Prematurity in Extremely Low Birth Weight Infants
}

Jihan Esmail, MS-2; Ramasubbareddy Dhanireddy, MD

The University of Tennessee Health Science Center College of Medicine, Memphis, TN, United States.

\section{Background}

* The association between hyperglycemia and ROP has been inconsistent in previous studies.

* Extremely low birth weight (ELBW) infants are at high risk for ROP. They often experience hypoglycemia initially and

hyperglycemia while receiving IV glucose infusion.

* The effect of initial hypoglycemia on hyperglycemia

associated $\mathrm{ROP}$ risk is unknown.

\section{Objective}

To study the effect of initial hypoglycemia and subsequent
hyperglycemia during the first three days of life on the incidence of ROP and severe ROP in ELBW (birth weight $\leq 1000 \mathrm{~g}$ ) infants.

\section{Methods}

Clinical and demographic data were collected from 224 ELBW infants born during the years 2017-2019 at the Regional One Health NICU, Memphis, TN.

All blood glucose determinations done during the first 72 hours were collected from these infants along with maternal and neonatal demographic and clinical information.

The infants were divided into four groups based on hypoglycemia at birth and subsequent hyperglycemia during the first 72 hours;

(25) blood glucose levels were between 47 125mg/al (euglycemia group);

Group II: Initial hypoglycemia (<47mg/dl) and with treatment - Gecame euglycemic,

作 hyperglycemia (>125mg/dl) subsequently

followed by at least one episode

Incidence of ROP and severe ROP (stage III or greater) was A age.

\section{Results}

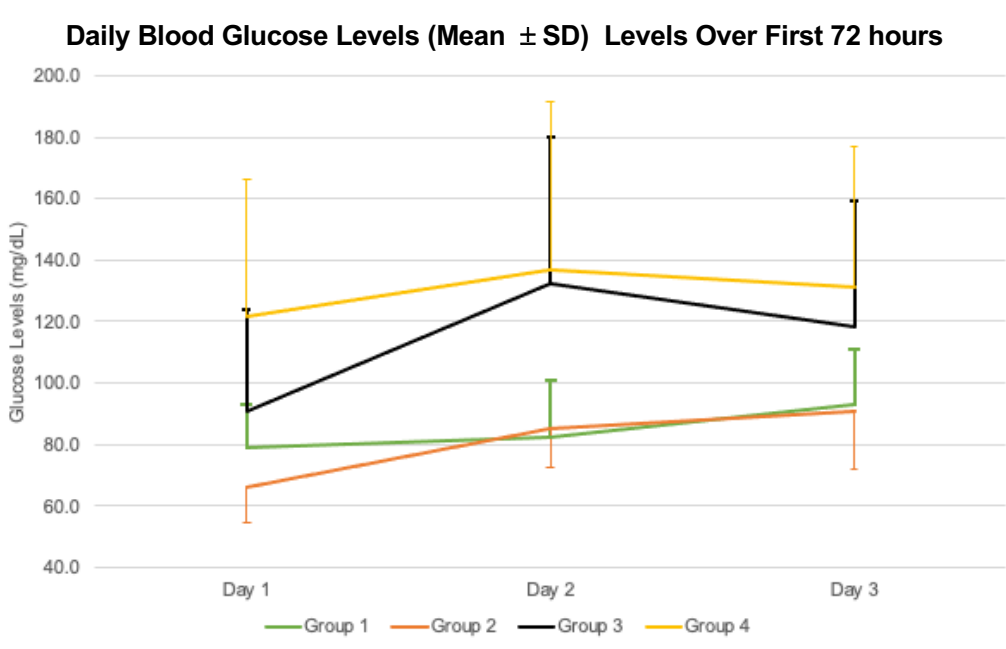

Blood glucose level and ROP in ELBW infants

\begin{tabular}{|c|c|c|c|c|c|c|c|c|}
\hline \multirow[b]{2}{*}{ Group $(n=224)$} & \multirow[b]{2}{*}{$\begin{array}{c}\text { GA } \\
\text { Mean } \pm \text { SD (weeks) }\end{array}$} & \multirow[b]{2}{*}{$\begin{array}{l}\text { Initial blood } \\
\text { glucose } \\
\text { Mean } \pm \text { SD } \\
(\mathrm{mg} / \mathrm{dL})\end{array}$} & \multicolumn{2}{|c|}{ Max Glucose level in first 72 hours } & \multicolumn{2}{|r|}{ ROP } & \multirow[b]{2}{*}{$\begin{array}{c}\text { Severe ROP } \\
(\%)\end{array}$} & \multirow[b]{2}{*}{$\begin{array}{l}\text { Mortality } \\
\mathrm{n}(\%)\end{array}$} \\
\hline & & & $\begin{array}{l}\text { Mean } \pm \text { SD } \\
(\mathrm{mg} / \mathrm{dL})\end{array}$ & $\begin{array}{l}\text { Median (Range) } \\
(\mathrm{mg} / \mathrm{dL})\end{array}$ & $n(\%)$ & $\begin{array}{c}\mathrm{aOR} \\
(95 \% \mathrm{Cl})\end{array}$ & & \\
\hline Group $1(28)$ & $26.6 \pm 2.3^{*}$ & $65 \pm 12.4$ & $103 \pm 16.1$ & $107(53-122)$ & $7(25 \%)$ & 1.0 & $0(0 \%)$ & $3(11 \%)$ \\
\hline Group 2 (33) & $27.5 \pm 2.5 \S$ & $33 \pm 9.5$ & $109 \pm 12.3$ & $110(84-125)$ & $15(45 \%)$ & $3.1(0.98-9.98)$ & $2(6 \%)$ & $4(12 \%)$ \\
\hline Group 3 (78) & $26.5 \pm 2.5 \dagger$ & $30 \pm 9.4$ & $205 \pm 75.6$ & $187(126-620)$ & $34(44 \%)$ & $2.3(0.87-6.08)$ & $9(12 \%)$ & $16(21 \%)$ \\
\hline Group 4 (85) & $25.3 \pm 1.9 * \S \dagger$ & $69 \pm 20.2$ & $212 \pm 93.1$ & $184(129-727)$ & $46(54 \%)$ & $3.6(1.33-9.68)$ & $12(14 \%)$ & $17(20 \%)$ \\
\hline
\end{tabular}

* ROP incidence was lowest in infants who were euglycemic throughout the first 72 hours of life and no severe ROP was

* Infants who were hypoglycemic initially without experiencing hyperglycemia later appear to have higher incidence of ROP though it was not statistically different after adjusting for

* Infants who were euglycemic at birth and became hyperglycemic later had increased incidence of ROP even fer adjusting for gestational age: they also had the highest incidence of severe ROP.

- Comparing combined groups 1 \&2 vs. 3 \&4 showed no difference in ROP [aOR 1.5 (082-2.84)]

Groups 182 vs $3 \& 4$ showed no difference in severe ROP OR $3.13(0.69-14.16)]$

* Mortality did not statistically differ between the groups.

\section{Conclusions}

Hyperglycemia during the first three days of life without hypoglycemia at birth increases the risk for ROP.

Hypoglycemia at birth appears to modify the risk of hyperglycemia associated ROP in ELBW infants.

ANOVA * $p=0.04 . \S p=0.0001 .+p=0.0043$. ROP aOR adjusted for gestational age 